\title{
Interaction Between CME and Magnetosphere Observed by Cluster on Nov. 6, 2001: (1) Waves Excitation
}

\author{
C. J. Xiao ${ }^{1}$, L. T. Song ${ }^{1,2}$, Z. Y. Pu ${ }^{2,1}$ J. X. Wang ${ }^{1}$, Z. X. Liu ${ }^{3}$, \\ K.-H. Glassmeier ${ }^{4}$, A. Balogh ${ }^{5}$, and H. Reme ${ }^{6}$ \\ ${ }^{1}$ National Astronomical Observatories, Chinese Academy of Sciences, Beijing 100012, China \\ email: cjxiao@pku.edu.cn \\ ${ }^{2}$ Institute of Space Physics and Applied Technology, Peking University,Beijing 100871, China \\ ${ }^{3}$ CSSAR, Chinese Academy of Sciences, Beijing 100080, China \\ ${ }^{4}$ IGEP, Technische Universität Braunschweig, Germany \\ ${ }^{5}$ Space and Atmospheric Physics Group, Imperial College, London, UK \\ ${ }^{6}$ CESR, BP 4346, 31028 Toulouse cedex 4, France
}

\begin{abstract}
Very few in situ measurements so far show the details of the interactions between CME and magnetosphere. Here we report a fortuitous observation that the Cluster spacecraft were located at the dusk-side magnetopause, meandering back and forth four times between the ICME and the magnetosphere, and observed part process of the interaction between the CME and the magnetosphere. The primarily analysis showed that: (1) a fast ion beam other than the ambient plasma was observed almost perpendicular to the magnetic field; (2) The left-handpolarized kinetic Alfvén waves excitation by the ion beam; and (3) the resonances between the kinetic Alfvén waves and part of the beam particles lead to the pitch angle diffusion of resonant particles and formating of a monospheric distribution with nearly constant kinetic energy. The wave-particle resonance may pay an important role in the energy transfer and particle exchange across the magnetopause between the magnetosheath and magnetosphere during the CME impulsion.
\end{abstract}

\section{Introduction}

Strong geomagnetic storm, usually caused by the CME-magnetosphere interaction, is one of the important parts of space weather(e.g., Gonzalez et al. 1994, Webb et al. 2000). Most researches focus on the relationship between the CME and the storm level of geomagnetic field(e.g., Wu and Lepping 2002, Russell and Mulligan 2002, Yermolaev et al. 2005 and references therein). There are so many spacecraft crossed through the magnetopause, but very few pass just be at the moment that the CME just arrived and began to compress the magnetosphere. so far there are too few in situ measurements to show the details of physical processes of the interactions between the CME with the front shock and magnetosphere (e.g., Huttunen et al. 2005).

The four-spacecraft Cluster mission (Escoubet et al. 1997) is designed to study the small-scale structures in key regions of the magnetosphere to provide the first opportunity to determine three-dimensional and time-dependent characteristics of small-scale processes in both the magnetosphere and nearby interplanetary medium (e.g., Hasegawa et al. 2004, Phan, et al. 2005). Here we report a fortuitous observation that the Cluster constellation located in such a interaction region between the CME and the magnetosphere. The magnetic field (B) of the FGM (Balogh et al. 1997), the plasma velocity (V) and particle distributions data of the CIS (Remé et al. 1997) onboard Cluster detected 

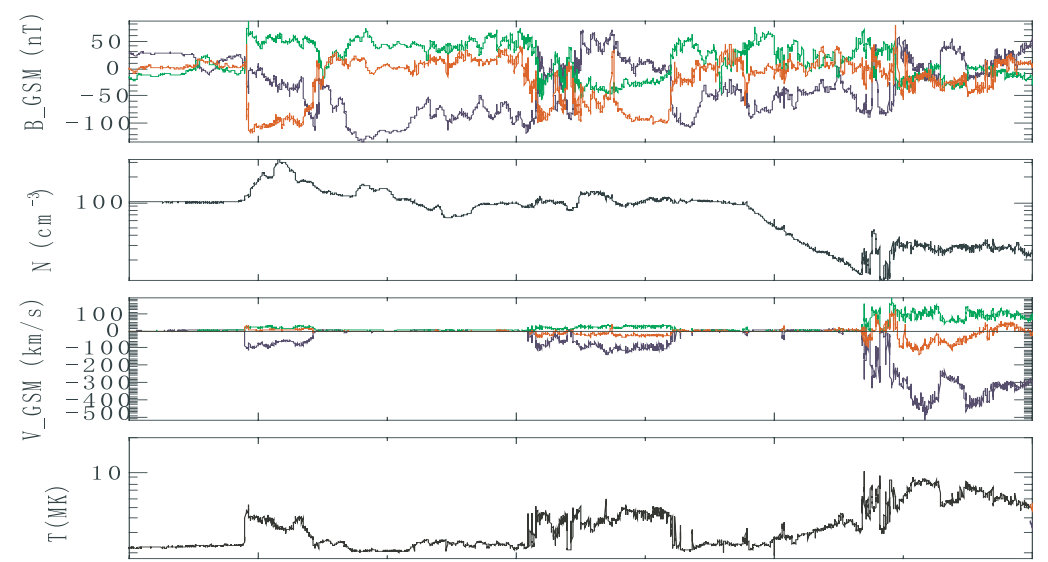

\section{Z_GSM \\ Y_GSM \\ X_G S M}

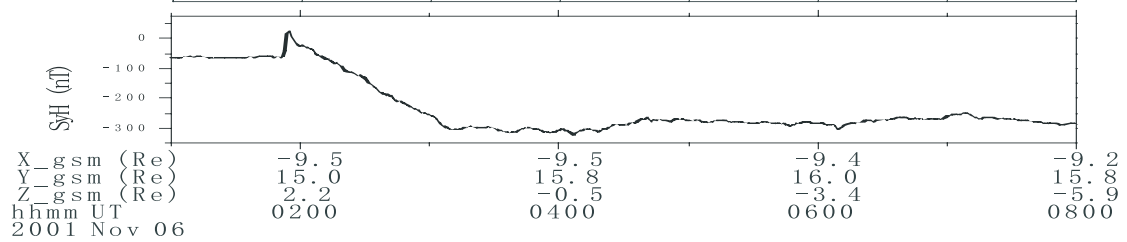

Figure 1. Overview of 4s resolution Cluster SC3 data and the 1-minute resolution symmetric disturbance index of $\mathrm{H}$ component of geomagnetic field $(\mathrm{SyH})$ during 01:00-08:00 UT on 6 Nov. 2001. From top to bottom are: the magnetic field (B), the density (N) and velocity (V) and temperature (T) of plasma, the Geomagnetic SyH component and the SC3 positions in GSM coordinates. The plots of the SyH data shows that the ICME began to compress the magnetosphere at 01:53 UT.

part phyiscal processes of interaction during the interval of the CME-magnetopause interactions.

\section{Observation Data Analysis}

An earth-direction halo CME was observed by LASCO/SOHO at 16:35 UT on 4 November 2001. Associated with the CME were a front shock and a magnetic cloud, which caused a strong magnetic storm with $D_{s t}<-280 n T$ during 6-8 November 2001. During the interval from 01:00 to 15:00 UT on 6 November 2001, the Cluster spacecraft located in the near-tail dusk magnetopause and meandered back and forth four times between the ICME and the magnetosphere. Overview of Cluster SC3 data and the geomagnetic disturbance index during 01:00-08:00 UT on 6 Nov. 2001 are shown in Figure 1. The rapid increase of the symmetric disturbance index of $\mathrm{H}$ component of geomagnetic field $(\mathrm{SyH})$ at 01:53 UT (the bottom panel in Figure 1) indicated that the CME with a front shock began to compress the magnetopause. Locating in the flank of the magnetopause at $(-9.6,15.0,2.2) R_{E}$ in GSM coordinates at 01:54 UT, the Cluster spacecraft outbound cross the magnetopause for the HIA velocities of SC3 increased from $(-8,-2,-2) \mathrm{km} / \mathrm{s}$ at 01:54:08 UT to $(-93,11,26) \mathrm{km} / \mathrm{s}$ at 01:54:16 UT. The amplitude of the magnetic field (FGM) jumped from $\sim 20 n T$ to $\sim 110 n T$, as well as the other plasma parameters, such as density and temperature, also increased sharply at the same time. In this event the Cluster SC3 kept staying in the ICME in about 31 minutes, then inbound cross the magnetopause at 02:27 UT (shown in Figure 1). The other inbound and outbound intervals are 04:08-05:14 UT, 06:40-09:53 UT and 10:05-11:30 UT (not shown here). There are lots of interesting physical processes in this event. As the first 


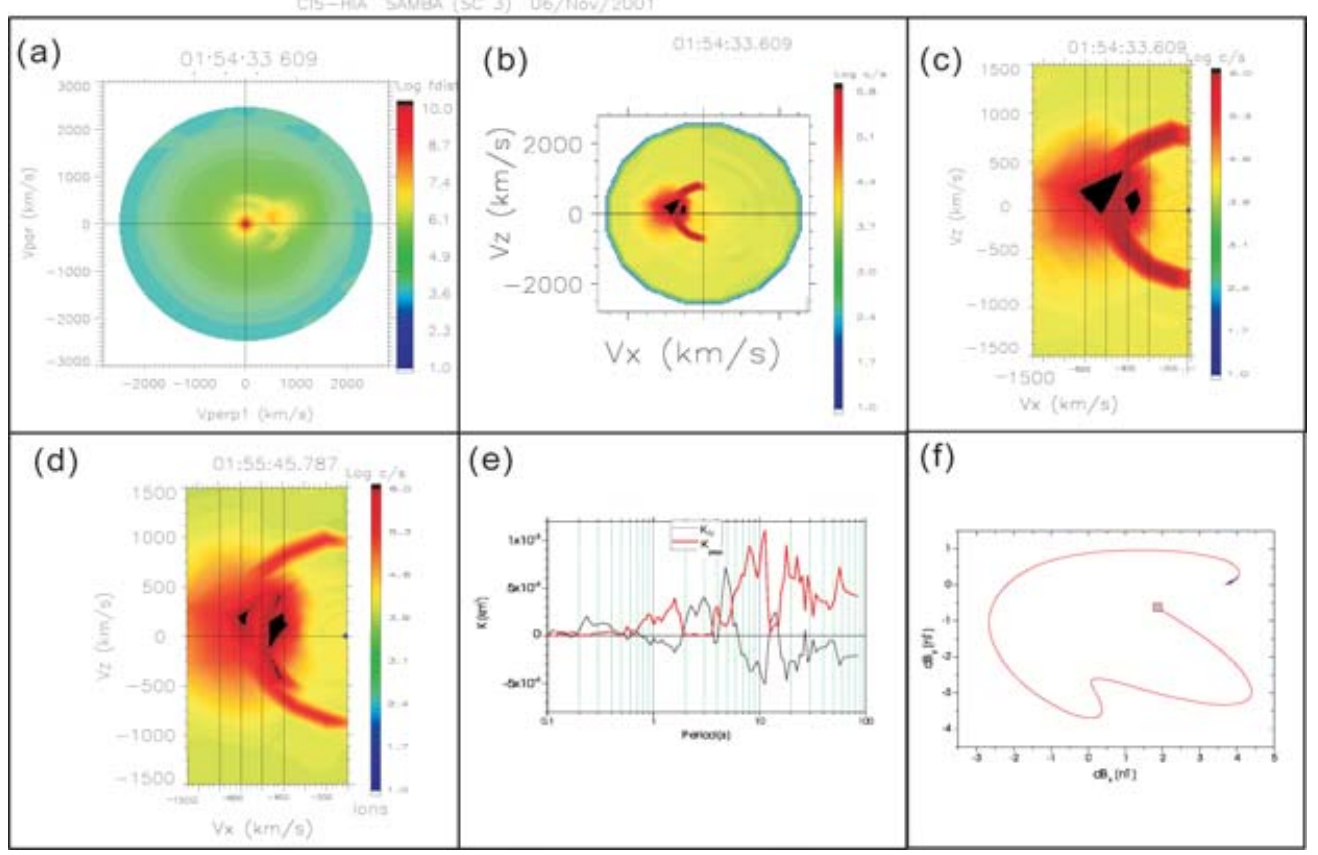

Figure 2. Ion distributions of SC3 at 01:54:33UT (a-c) and 01:55:45UT (d). (a)Ion distribution in $V_{/ /}-V_{\perp}$ plane at 01:54:33UT; (b)Ion distribution in GSE $V_{x}-V_{z}$ plane at 01:54:33UT; (c)Amplifying and rescalling the ion distribution shown in (a); (d)Ion distribution in GSE $V_{x}-V_{z}$ plane at 01:55:45UT; (e)the relationship between the wave vectors and periods; (f)Polarization of $(\mathrm{dBx}, \mathrm{dBz})$, bandpass-filtered data at $10-20$ s periods. The start point marked with square.

part of work, in the following part we focus on the data analysis of waves excitation and wave-particle resonances during the first travel outbound the magnetopause form 01:54 to $02: 27 \mathrm{UT}$.

Based on 0.04s high-resolution FGM data (not shown here for the limit of paper length)we found that all 4 Cluster spacecraft crossed the current sheet of magnetopause during 01:54:25-01:54:30UT. After checking the ion distribution data of CIS onboard SC3 it is also found that a fast ion beam identified clearly from the ambient plasma was observed between 01:54:21UT and 02:23:01UT. The ion beam is almost perpendicular to the magnetic field. It is a conventional point of view that nonlinear wave are excited as a result of ion beam instability (Galinsky et al. 1997, Shevchenko et al. 2002). As a result of the wave excitation, the pitch angle diffusion of resonant particles lead to formation of a monospheric distribution function with constant kinetic energy (see Lee 1989). They were observed near the Earth's bow shock (Russell and Hoppe 1983) and upstream of the shock near the other planets or comets (Tsurutani et al. 1995). In this event, during 01:54:33-01:58:55UT, it is found that the pitch angle diffusions of resonant particles were observed near the magnetopause, which far from any shock wave as previous cases.

As a the example, the ion distribution of SC3 in the $V_{/ /}-V_{\perp}$ phase space at 01:54:33UT is shown in Figure 2a. The pitch angle diffusion of resonant ions lead to formation of a monospheric distribution with constant kinetic energy $\left(V_{/ /}^{2}+V_{\perp}^{2} \simeq\right.$ Const.). Figure $2 \mathrm{~b}$ showed the monospheric distribution clearly as that shape of a half circle in $V_{x}-V_{z}$ phase space, for the z-direction parallel to B. As enlarged the $V_{x}<0$ part of Figure 2b, it is clearly in Figure 2c that the diffusion ions consisted in two arcs: one changes from $V_{x} \sim-700 \mathrm{~km} / \mathrm{s}$ into $V_{z} \sim-700 \mathrm{~km} / \mathrm{s}$; the other $V_{x} \sim 900 \mathrm{~km} / \mathrm{s}$ into $V_{z} \sim 900 \mathrm{~km} / \mathrm{s}$. 
Another monospheric ion distribution at 01:55:45UT is shown in Figure 2d, which also consisted with two $\operatorname{arcs}$ with $\left(V_{/ /}^{2}+V_{\perp}^{2}\right)$ close to $(900 \mathrm{~km} / \mathrm{s})^{2}$ and $(1100 \mathrm{~km} / \mathrm{s})^{2}$, higher kinetic energy than that at 01:54:33UT.

Theoretically these particle distributions usually took placed during the wave-particle resonance, even very few previous observations like so mono-energy that have been found. To identify the wave mode during the CME-magnetosphere interaction process, the best way is analysis with electric, magnetic fields and plasma data. But there is no good electric field data on that day. So now we used the methods of wavelet coherence analysis and cross wavelet transform (Grinsted et al. 2004, Chaston et al. 2005) on the 0.04s high resolution magnetic data, to find the relationship of the frequencies and wave vectors. The averaged results from 01:55:00-01:56:30UT are shown in Figure 2e. It is clearly that The bigger wave numbers happened with periods 8 -30s and $K_{\perp} \simeq 2 K_{/ /}$. The results of wavelet analysis (not shown here) also confirmed that the periods of the relative strong Wave mode is low-frequency (about 1-40s) during 01:54:30-01:57:00 UT. After bandpass filtering of $0.04 \mathrm{~s}$ FGM data at 10-20s periods (Sundkvist et al. 2005), the lefthand polarization properties of the low frequency waves are shown in Figure $2 \mathrm{f}$.

\section{Conclusions}

During the processes of CME-magnetosphere interactions, an ion beam was observed by the Cluster spacecraft at the magnetopause. The left-hand-polarized kinetic Alfvén waves excited by the ion beam can identified with the relationship of the frequencies and wave vectors. The resonances between the kinetic Alfvén waves and part of the beam particles lead to pitch angle diffusion of resonant particles and a monospheric ion distribution with constant kinetic energy, which matches the theory very well. More detailed calculations and analysis are needed for further analysis.

\section{Acknowledgements}

This work is supported by the NSFC Programs (Grant No. 40390150 and 4050421).

\section{References}

Balogh, A., Dunlop, M.W., Cowley, W. H., et al., 1997 Space Sci. Rev. 79, 65 Chaston, C.C., T.D. phan, J.W. Bonnell, et al., 2005 Phys. Rev. Lett. 95, 065002

Escoubet, C. P., R. Schmidt and M. L. Goldstein, 1997 Space Sci. Rev. 79, 11 Galinsky, V.L., V.I. Shevchenko, S.K. Ride and M. Braine, 1997, J. Geophy. Res. 102, 22365

Grinsted, A., S. Jevrejeva and J. Moore, 2004 Nonlinear Processes in Geophysics 11, 561

Gonzalez, W. D., J. A. Joselyn, Y. Kamide, et al., 1994, J. Geophy. Res. 99, 5771

Hasegawa, H., et al., 2004, Nature 430, 755

Huttunen, K. E. J., J. Slavin, M. Collier, et al., 2005, Annales. Geophy. 23, 609

Lee, M. A., 1989, in Magnetospheres, Geophys. Monogr. Ser., vol. 53, (ed.) B. Tsurutani and H. Oya, AGU, p41.

Phan, T. D., C. P. Escoubet, L. rezeau, et al. 2005, Space Sci. Rev. 118, 367

Remé, H., Bosqued, J. M., Sauvaud, J. A., et al., 1997 Space Sci. Rev. 79, 303

Russell, C. T. and M.M. Hoppe, 1983 Space Sci. Rev. 34, 155

Russell, C. T. and T. Mulligan, 2002, Planetary and Space Sci. 50, 527

Shevchenko, V.I., et al., 2002, J. Geophy. Res. 107, doi:10.1029/2001JA009028

Sundkvist, D., et al., 2005, Nature 436, doi:10.1038/nature03931

Tsurutani, B.T., et al., 1995, Geophys. Res. Lett. 22, 1149

Webb, D.F., et al., 2000, J. Geophy. Res. 105, 7491

Wu, C. C. and R. P. Lepping, 2002, J. Geophy. Res. 107, A4, doi:10.1029/2001JA000161

Yermolae, Yu I., et al., 2005, Planetary and Space Sci. 53, 189 